\title{
Les verbes à objet implicite dans le français parlé :
}

de la proéminence informationnelle à la proéminence phonologique

Verbs with implicit objects in spoken French: from informational prominence to phonological prominence

\section{Anouch Bourmayan}

\section{OpenEdition}

Journals

\section{Édition électronique}

URL : http://journals.openedition.org/tipa/1200

DOI : 10.4000/tipa. 1200

ISSN : 2264-7082

\section{Éditeur}

Laboratoire Parole et Langage

\section{Référence électronique}

Anouch Bourmayan, «Les verbes à objet implicite dans le français parlé : », TIPA. Travaux

interdisciplinaires sur la parole et le langage [En ligne], 30 | 2014, mis en ligne le 12 décembre 2014, consulté le 19 avril 2019. URL : http://journals.openedition.org/tipa/1200 ; DOI : 10.4000/tipa.1200

Ce document a été généré automatiquement le 19 avril 2019.

\section{(c) ()) (9)}

La revue TIPA. Travaux interdisciplinaires sur la parole et le langage est mise à disposition selon les termes de la licence Creative Commons Attribution - Pas d'Utilisation Commerciale - Pas de Modification 4.0 International. 


\title{
Les verbes à objet implicite dans le français parlé:
}

\author{
de la proéminence informationnelle à la proéminence phonologique \\ Verbs with implicit objects in spoken French: from informational prominence to \\ phonological prominence
}

\section{Anouch Bourmayan}

1 Un élément $\mathrm{X}$ est proéminent par rapport à un élément $\mathrm{Y}$ quand $\mathrm{X}$ est plus en relief, plus saillant que $Y$. Sur un plan phonologique, le degré extrême de la proéminence d'un item linguistique $\mathrm{X}$ par rapport à un autre item linguistique $\mathrm{Y}$ est le cas où $\mathrm{X}$ est réalisé phonologiquement et $\mathrm{Y}$ ne l'est pas, comme l'illustre par exemple le phénomène des verbes à objet implicite. En effet, certains verbes sont sémantiquement transitifs et requièrent donc nécessairement un objet sémantique, mais peuvent néanmoins apparaître sans complément d'objet direct phonologiquement réalisé : l'objet sémantique reste alors implicite. Ainsi, un verbe comme manger peut par exemple être utilisé sans complément d'objet explicite, comme en (1):

(1) Jean est en train de manger.

2 L'énoncé signifie alors que Jean mange quelque chose, une chose dont l'identité précise n'importe pas : manger est ici interprété avec un objet implicite indéfini (ou OII). Mais si je crie (2) en lançant une balle à quelqu'un,

(2) Attrape !

3 mon énoncé sera compris comme une injonction à attraper non pas une chose ou une autre, mais précisément la balle que je lance : attrape est alors interprété avec un objet implicite défini (ou OID). Cependant, d'autres verbes semblent a priori admettre plus difficilement les objets implicites. Ainsi, les énoncés (3) et (4) apparaissent par exemple difficilement recevables, l'omission de l'objet avec le verbe faire ne semble pas ici tolérée : 
(3) Marie est en train de faire.

(4) Fais !

4 plusieurs pour l'anglais - selon lesquelles la capacité d'un verbe à admettre les OIIs ou les OIDs constitue une propriété lexicale du verbe. Ainsi, Fillmore (1986) tout d'abord puis plus récemment Gillon (2012) ont affirmé qu'il s'agit d'une propriété fixée dans le lexique d'une manière purement arbitraire, qui ne peut être déduite des autres propriétés sémantiques du verbe. Mittwoch $(1971,1982)$ et Noailly (1998) quant à elles analysent plutôt la possibilité d'admettre des OIIs ou des OIDs comme le résultat des propriétés aspectuelles du verbe : ainsi, les verbes admettant les OIIs seraient des verbes atéliques, tandis que les verbes admettant les OIDs seraient téliques. Enfin, Rappaport Hovav et Levin (1998) développent une approche dite événementielle, selon laquelle les verbes causatifs n'admettent pas l'omission de l'objet du fait de leur structure événementielle complexe. Une limite évidente des approches lexicalistes est cependant qu'elles sont souvent basées sur des corpus écrits, voire sur des exemples inventés, qui courent ainsi le risque d'être biaisés, les derniers parce qu'ils sont fondés sur l'introspection, les premiers parce qu'ils reflètent un usage extrêmement contraint de la langue, qui peut ne représenter qu'imparfaitement le phénomène tel qu'il apparaît dans le français usuel, c'est-à-dire dans le français parlé. De fait, comme l'affirment Lambrecht \& Lemoine (2005) :

6 [Le phénomène] échappe dans une grande mesure à la capacité du locuteur natif à formuler des jugements d'acceptabilité indépendamment de contextes discursifs donnés. Nous avons ici affaire à un domaine de la syntaxe du français contemporain auquel l'introspection fournit seulement un accès limité. L'utilisation de corpus de production orale spontanée s'avère ainsi essentielle dans ce champ. ${ }^{1}$

Bien que Lambrecht \& Lemoine ne fassent cette affirmation qu'à propos d'un type donné d'objets implicites, les OIDs dits "topiques", celle-ci peut en fait être considérée dans une certaine mesure comme pertinente pour tous les types d'objets implicites. C'est sur ce postulat d'une primauté des corpus oraux par rapport au français écrit et à plus forte raison par rapport aux données introspectives que j'appuierai ici l'ensemble de ma démonstration.

8 Pour ce faire, je me fonde ici spécifiquement sur le Corpus de Français Parlé Parisien des années 2000 ou CFPP 2000 (Branca-Rosoff, Fleury, Lefeuvre et Pires 2009), dont le prétexte est d'interroger les habitants de Paris et des quartiers limitrophes sur leur rapport à leur quartier. Pour opérer le choix des occurrences de verbes comptant un objet implicite dans ce corpus, mes critères ont été les suivants. Tout d'abord, j'ai laissé de côté les verbes présentant un objet sémantique syntaxiquement détaché en périphérie de la proposition, et non repris par un complément d'objet direct explicite, comme en $(5)^{2}$ :

(5) a. tu vois l'photographe à côté je fais travailler bon la presse elle est à côté

b. mais c'est vrai que cinquième sixième septième j'connais bien et j'aime beaucoup

c. euh alors la Pyramide $+\mathrm{j}$ 'suis pas $+\mathrm{j}$ 'ai jamais $\underline{\mathrm{vu}}$ avant en $\mathrm{fait}^{3}$

d. le Carrousel les lumières euh + j'aime beaucoup 
e. le quartier populaire euh + du donc du nord du de l'est + + vous connaissez un petit peu

f. l'automobiliste on taxe déjà beaucoup

En effet, chacune des occurrences de cette construction soulève la question de savoir si l'on a affaire à une structure de topicalisation syntaxique au sens strict, c'est-à-dire telle que le complément d'objet direct a subi un mouvement syntaxique de sa position initiale vers une position de topique en lieu et place du complémenteur, d'où l'absence de complément d'objet direct explicite aux côtés du verbe, ou bien s'il s'agit d'un constituant détaché, qui a donc été simplement adjoint à la proposition et n'est pas repris par un complément d'objet direct explicite. Or dans le premier cas, il pourrait être argué qu'il s'agit d'une construction syntaxique tout à fait spécifique, qui n'implique pas d'objet implicite au sens strict, puisque le complément d'objet - explicite - a tout simplement été déplacé plus haut dans la proposition.

Je laisserai également de côté les cas où l'objet implicite est paraphrasable par un pronom se situant aux côtés d'un autre pronom. Ainsi, en (6), la proposition finale et j'lui commande est paraphrasable par et je la lui commande :

(6) Sarah enq : vous savez nous + pour ces mêmes raisons on + en venant habiter là on a décidé d'acheter

Pauline de Bordes : c'est très

Sarah enq : nos livres chez le libraire du coin

Pauline de Bordes : ben voilà

Sarah enq : pour qu'il puisse tenir le coup justement

Pauline de Bordes : voilà

Sarah enq : donc c'est c'est ça maintenant mon libraire

ah oui c'est ça et on fait c'est et moi j'fais les listes et j'lui commande

Or on pourrait arguer que la disparition du pronom objet la est le fruit d'un phénomène phonologique classique d'haplologie, tel que la proximité phonologique de deux phonèmes ou groupes de phonèmes, en l'occurrence les pronoms la et lui, entraîne la disparition de l'un d'entre eux. Ainsi, pour montrer que la distribution des objets implicites ne se réduit pas à des contraintes phonologiques et que l'haplologie est en fait un cas tout à fait particulier, je laisserai de côté les occurrences de verbes apparaissant aux côtés d'un pronom susceptible d'avoir provoqué la disparition phonologique du complément d'objet direct comme (6).

Une autre difficulté qui se pose dans un corpus de français parlé est celle de savoir dans quels cas le locuteur a exprimé une proposition complète et a donc fait le choix de laisser l'objet sémantique implicite, et dans quel cas il a simplement interrompu son propos après le verbe, pour une raison ou pour une autre. Afin de trancher, un certain nombre d'indices peuvent être utilisés, parmi lesquels des indices textuels, mais également des indices sonores, puisqu'un enregistrement de l'enquête est fourni avec le texte du CFPP 2000. Ainsi, dans l'énoncé en (7), prononcé dans le contexte d'une discussion portant sur les chiens et par lequel l'enquêteuse Florence s'enquiert du fait que Lucie, la personne interrogée, sorte ou non avec ses chiens quand elle va faire ses courses, la présence de l'interjection euh est un premier indice que le locuteur a interrompu son discours. En outre, une audition de la bande sonore correspondante permet d'entendre que Lucie, 
immédiatement après l'interjection de Florence, a interrompu cette dernière, ne lui laissant pas le loisir d'ajouter un éventuel complément d'objet direct au verbe prendre:

(7) Florence Enq : est-ce que pour aller faire des courses par exemple vous ça vous arrive de prendre euh :

Lucie : alors

(8) c'est possible mais dans le métro on voit moins parce qu'on voyait effectivement des rassemblements dans le métro

5 Faut-il considérer la première occurrence de voir comme un cas de verbe à objet implicite? A nouveau, il semble plus prudent d'écarter ce type d'occurrence, dans la mesure où le risque est fort que le locuteur ait simplement souhaité reformuler son propos, et que la première occurrence du verbe ne corresponde ainsi pas à une prédication complète, dans laquelle le locuteur aurait souhaité garder l'objet implicite.

A partir des données extraites du CFPP 2000 sur la base de ces critères, je montrerai dans une première section que les approches lexicalistes sont erronées pour le français parlé, et qu'il n'est pas vrai que l'omission de l'objet y est déterminée par les caractéristiques lexicales du verbe. Puis dans une seconde section, je présenterai une analyse originale du phénomène selon laquelle l'omission de l'objet est guidée par des critères d'ordre communicationnel, selon le principe suivant :

7 La proéminence phonologique du verbe sur l'objet sémantique (c'est-à-dire la réalisation phonologique $\mathrm{du}$ verbe et la non-réalisation de l'objet sémantique) traduit une proéminence d'ordre informationnelle: un objet sémantique est laissé implicite si et seulement si l'action décrite par le verbe est plus saillante que l'objet sémantique sur un plan informationnel.

8 A partir du corpus, nous nous pencherons sur trois types d'objets implicites différents, les OIDs pronominalisables, les OIDs non pronominalisables, et les OIIs, et nous verrons que chacun de ces types d'objets implicites, avec ses caractéristiques propres, conforte la thèse ci-dessus, selon laquelle la proéminence phonologique du verbe par rapport à l'objet sémantique est fonction de sa proéminence informationnelle.

\section{Les approches lexicalistes à l'épreuve du corpus}

19 Si plusieurs analyses ont été proposées pour rendre compte de la distribution des objets implicites en français, une littérature très importante existe sur le sujet concernant la langue anglaise, à laquelle je ne m'interdirai pas ici de faire référence, du fait de son ampleur et de la proximité apparente des deux langues. Or qu'il s'agisse de l'anglais ou du français, les approches formulées sont majoritairement de nature lexicaliste, au sens où elles situent la capacité d'un verbe donné à admettre ou non les objets implicites, et plus 
spécifiquement les OIIs ou les OIDs, à un niveau lexical; en d'autres termes, la capacité à admettre les objets implicites serait une propriété des types de verbes et non simplement de leurs occurrences, chaque item verbal encodant dans son entrée lexicale la capacité ou l'incapacité à admettre les OIIs ou les OIDs. Au sein de cette approche lexicaliste, plusieurs analyses ont été formulées, qui diffèrent quant aux critères lexicaux précis permettant à un verbe d'admettre les objets implicites.

\subsection{L'approche lexicaliste arbitraire}

Selon une première analyse lexicaliste, la capacité d'un verbe à admettre les objets implicites - OIIs ou OIDs - est fixée dans le lexique d'une manière tout à fait arbitraire, qui ne peut être déduite d'autres propriétés sémantiques du verbe. C'est par exemple l'hypothèse défendue par Fillmore (1986), et plus récemment par Gillon (2012) pour l'anglais. Noailly (1994) adopte pour certains verbes un point de vue similaire pour le français, affirmant par exemple qu'un verbe comme faire est réfractaire à l'emploi absolu, c'est-à-dire à l'emploi avec objet implicite. Or notre corpus montre qu'il n'en est rien, qu'un verbe comme faire peut être employé avec un objet implicite dans certains contextes. En témoigne notamment l'échange en (9), où l'on comprend que l'objet implicite de faire correspond aux paëllas :

(9) Enq SB : vous faites des paëllas

Etienne : non plus

Ozgur : pas vraiment

Etienne : (non, nous) on les mange mais on les fait pas

Enq $\mathrm{SB}$ : vous mangez mais vous faites pas

L'on pourrait cependant arguer que l'acceptabilité de l'objet implicite est ici favorisée par le contexte syntaxique, contrastif d'une part, puisque le verbe faire est opposé au verbe manger, mais également habituel. De fait, Noailly comme d'autres linguistes admet que certains contextes syntaxiques favorisent l'acceptabilité des objets implicites, et prétend que ces facteurs syntaxiques permettent de surmonter les tendances lexicales du verbe. Or notre corpus montre que faire peut apparaître avec un objet implicite même dans des contextes non contrastifs et non habituels, comme en témoigne l'exemple (10):

(10) alors j'ai eu cette expérience puis j'ai un peu travaillé aussi pour euh donc aller dans la rue voir les donc euh $j$ 'ai fait pendant un an

Ici, l'objet implicite est évidemment paraphrasable par le pronom ça, et correspond sémantiquement au travail décrit par la locutrice dans la première partie de son énoncé.

De même, Noailly (1998) affirme qu'un verbe comme habiter n'admet pas l'omission de son complément d'objet direct. Mais là encore, le corpus semble contredire cette affirmation. L'échange en (11) peut tout d'abord être interprété comme une première illustration de la capacité d'habiter à être employé avec un objet implicite, quoique cet exemple puisse être dans une certaine mesure sujet à caution :

(11) Pauline de Bordes : heureusement qu'y avait les associations qui + eux font un travail de fond parce que ça donne c'est pas si facile que ça de mettre des gens dans un local hein c'est c'est pas vrai malheureusement c'est pas vrai 
Sarah enq : oui ceux qui sont très abimés par la vie ne savent plus

Sarah enq : habiter

Pauline de Bordes : mais ils savent

Sarah enq : ils savent hein donc il faut un accompagnement heu très très euh

Pauline de Bordes : mais non ils n'ont ils n'savent plus habi- mais oui qui dure quelquefois.

Certes, on peut douter du fait que l'enquêteuse Sarah ait vraiment énoncé une proposition complète lorsqu'elle emploie le verbe habiter sans complément d'objet. Mais cette hypothèse me semble néanmoins loin d'être absurde, car ce qui est en jeu ici est bien la capacité d'habiter en elle-même, c'est-à-dire celle de prendre possession d'un lieu, de l'investir de manière autonome, effort qui pour les personnes marginalisées évoquées par les deux locutrices peut s'avérer considérable. Un autre exemple, illustrant plus clairement l'emploi absolu d'habiter, est l'énoncé en (12):

(12) en même temps c'est vrai qu' c'est un quartier assez tranquille entre guillemets c'est-à-dire qu'au niveau d' la vie nocturne on n'a pas vraiment de nuisances + comparé à d'autres quartiers comme le Quartier Latin par exemple qui est aussi agréable mais bon c'est pas du tout j' le vois pas pareil pour habiter j' trouve ça un petit peu ++ un petit peu moins agréable ++

Enfin, un emploi absolu évident d'habiter, que le corpus a simplement le mérite de nous remettre en mémoire mais auquel tout un chacun a déjà été confronté, se trouve dans l'expression habiter seul, comme en (13):

(13) donc euh nous on é- on était grands à partir de seize ans quoi $(\mathrm{mm})$ j'suis venu en France à seize ans + $(\mathrm{mm})+$ donc de l'adolescence on passe directement à la vie de grand parce que il faut habiter tout seul il faut préparer à manger il faut trouver du travail

De fait, habiter peut ici être interprété avec un OII au sémantisme non restreint, paraphrasable par un logement, parce la valeur précise de l'objet sémantique n'importe aucunement: ce qui est en jeu est bien l'activité elle-même décrite par habiter, et plus précisément la manière dont celle-ci est accomplie, indépendamment de l'objet précis auquel elle s'applique.

Ainsi, le CFPP 2000 permet de relativiser la thèse selon laquelle la capacité d'un verbe à admettre les objets implicites est fixée arbitrairement dans le lexique. En outre, le corpus montre également qu'il est erroné de considérer certains verbes comme n'admettant que les OIIs, et d'autres comme n'admettant que les OIDs. Pour n'en donner qu'un exemple, considérons le verbe lire. Il a été affirmé à maintes reprises dans la littérature à propos de lire et à propos de son homologue anglais read (voir Fillmore 1986, Gillon 2012, Martí 2009 ou encore Noailly 1997) que le verbe n'admettrait lexicalement que les OIIs, et non les OIDs. Le CFPP 2000 montre qu'il n'en est rien, comme il apparaît en (14) :

(14) a. Bernard : ah oui je lis volontiers l'journal du quartier Bernard : enfin je regarde ça comme ça d'un oeil un peu truc Micheline : je décortique quand il y a des trucs dans la rue je lis j'me mets au courant b. enq FL : non + + peut-être les journaux euh vendus librement là enfin qui qui 
coûtent rien

Gary COLLARD : euh beh pff j'en ai jamais à ma station je + je je lis très rarement c. j-j'ai pas j'ai pas cet envie d'un envie particulière + + de $m^{\prime}$ renseigner j' reçois de temps en temps un + un journal du vingtième mais bon c'est très rare $(\mathrm{mm})+\mathrm{c}^{\prime}$ est pas du tout régulier je + si un jour j'en ai dans ma boîte aux lettres bon ben okay + puis j'ouvre je lis

d. Killian : y a un le Kremlinois c'est un journal local

Lucas : oui Enq. Sarah : le kremlinois oui

Killian : c'est un journal oui qui parle du kremlin

Enq. Sarah : vous le lisez?

Killian : j'le feuillette

Enq. Sarah : et il est distribué gratuitement?

Lucas : oui

Killian : oui euh

Lucas : oui dans les boîtes aux lettres oui

Enq. Sarah : donc euh tout y compris nécrologie euh

Killian : ah non

Lucas : non j'sais que ++ mes parents lisent un petit peu moi non moi jamais mais mes parents qui sont là depuis longtemps

Killian : non moi c'est plutôt pour les informations pratiques

Lucas : oui pareil «journaux [...] vendus librement », en (14c) à un exemplaire du journal du vingtième, et en (14d) au Kremlinois. Ainsi, le CFPP 2000 montre bien que la capacité d'un verbe à admettre les objets implicites, et plus spécifiquement les OIIs ou les OIDs, n'est pas fixée de manière définitive dans le lexique, mais dépend largement du contexte d'utilisation du verbe, un fait qui n'apparaît pas nécessairement à la seule introspection, mais est nettement visible dans un corpus comme CFPP 2000 correspondant à une production orale et spontanée.

\subsection{L'approche lexicaliste aspectuelle}

première limite de cette approche, immédiatement visible, est qu'elle n'est pas exhaustive. En effet, si l'on sait que les verbes admettant les OIIs sont atéliques et les verbes admettant les OIDs téliques, il n'est pas dit que tous les verbes atéliques admettent les OIIs, ni que tous les verbes téliques admettent les OIDs. Ainsi, quels verbes parmi les atéliques admettent les OIIs, et quels verbes parmi les téliques admettent les OIDs ? Cette approche ne permet pas de le déterminer.

Cependant, l'hypothèse initiale selon laquelle seuls les verbes atéliques pourraient admettre les OIIs, tandis que seuls les verbes téliques admettraient les OIDs, est-elle ellemême confirmée? Rien n'est moins sûr, si l'on considère le CFPP 2000. En effet l'on trouve tout d'abord dans le corpus plusieurs exemples de verbes intrinsèquement 
téliques, c'est-à-dire dont l'action présente nécessairement un point d'aboutissement, utilisés avec des OIIs. En (15) par exemple, le verbe télique acheter est interprété avec un OII paraphrasable par des produits alimentaires :

(15) moi j'vois les prix qui augmentent mais bon faut bien manger donc j'achète hein également beaucoup question d'immobilier, et les verbes téliques acheter et racheter apparaissent ainsi également à maintes reprises avec un OII paraphrasable par un appartement/des appartements, comme en témoignent notamment les exemples en (16) :

(16) a. y a énormément d'Chinois qui arrivent énormément + $(\mathrm{mm}) \mathrm{d}$ 'chinois qui rachètent (et en c'moment énormément d'Chinois qui revendent parce que le quartier a est en train d'monter)

b. je pense effectivement que à Belleville se passe aujourd'hui le même processus c'est-à-dire qu'ils ont énormément racheté ils ils achètent beaucoup moins aujourd'hui ils vendent ils vendent à qui à des personnes qui ont beaucoup plus d'argent donc les bobos

c. on dit que les Italiens achètent beaucoup dans Paris ?

d. euh y a pas mal de + de + parents qui achètent pour leurs enfants étudiants + e. donc euh : c'est vrai qu' c'est ça qu'à mon avis qu'a fait que l'quartier est très catrès très ca(lme) très très très calme à l'époque + et en fait on voit de plus en plus de jeune qu'arrivent vers ce quartier là qui emménagent qui achètent euh pour euh : des jeunes mariés avec leurs leurs enfantsf. Francine : j'les trouve mal barrées euh pour acheter

g. nous avons acheté ici parce que nous considérions que pour pour vieillir nous étions mieux dans un appartement qui nous appartenait que dans un appartement de location

h. et on peut pas acheter dans Paris

i. alors voilà + la population s' transforme pour ça + + la rue d' Paris s' transforme ++ il y a la B.N.P. qui s'est installée + des grosses entreprises qui s' sont installées + il y a Air-France qui s'est installée + + vous voyez + + la ville change + + alors elle change en + en f-comment dirai-je en sélectionnant la population par l'argent en gros + les gens qui achètent maintenant ben il faut qu'ils aient des revenus hein quand même hein

Enfin, on peut trouver également d'autres verbes téliques comme réparer, apporter ou encore donner avec des OIIs :

(17) a. donc aussi bien moi j'me souviens euh euh des orfè- des des bijoutiers qui faisaient un travail d'orfèvrerie y avait des matelassiers y avait des gens qui réparaient aussi donc des artisans d'art hein

b. Isabelle : ben c'est en fait on s' réunit euh euh sur la petite place là qu'est juste à côté là où y $\mathrm{ala}+(\mathrm{mm}) \mathrm{l}$ 'horloge on va dire et en fait c'est des tables qu'on met dehors et $(\mathrm{mm})$ euh

Enq.Sonia : et les gens voilà

Isabelle : et chacun apporte

Isabelle : viennent manger $(\mathrm{mm})$ et apportent +

c. mon père m'a toujours dit " $t$ 'as besoin d'argent $j$ 'te donne" mais jamais voulu euh 
Les OIIs sont alors respectivement paraphrasables par des objets cassés, de la nourriture, et de l'argent.

A l'inverse, le CFPP 2000 met également en évidence des verbes atéliques employés avec un OID. Ainsi, en (18), travailler, resserrer, commenter, garder et suivre sont tous employés avec un OID :

(18) a. moi je vois qu' par exemple par rapport à mes élèves de piano la plupart maintenant travaillent sur du numérique + parce que en termes de voisinage euhb. oui oui + absolument + euh + je + je vous ai pas demandé alors + si je continue à

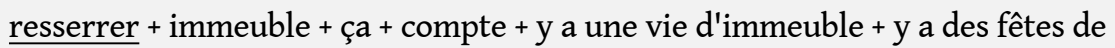
voisins?

c. Sarah en : on n'entend pas les gens commencer à commenter le prix des choses? Yvette : ah mais les gens ont toujours commenté ici + ça c'est la différence d. A propos des dépenses effectuées par les parents pour acheter des marques à leurs enfants : et ça va les mettre très mal à l'aise au bout d'un moment + ils pourront pas suivre e. SBenq : les cours d'ELCO vous avez suivi Youcef : non moi j'ai pas suivi

36 En (18a), l'OID de travailler est paraphrasable par le piano, celui de resserrer en (18b) par le thème de la conversation et l'OID de commenter en (18c) correspond au prix des choses évoqué précédemment, tandis que les OIDs de suivre en (18d) et (18e) sont respectivement paraphrasables par le rythme des dépenses pour les marques et par les cours d'ELCO 4 .

Un examen attentif des données du CFPP 2000 montre ainsi que l'approche lexicaliste aspectuelle ne parvient pas à rendre compte de manière satisfaisante de l'omission de l'objet dans le français parlé. Qu'en est-il de la dernière approche lexicaliste, l'approche lexicaliste événementielle?

\subsection{L'approche lexicaliste événementielle}

Une dernière approche lexicaliste qui a été défendue dans la littérature est l'approche dite événementielle de Rappaport Hovav et Levin (1998). Pour la résumer, selon cette analyse, les verbes causatifs n'admettent pas l'omission de l'objet en raison de leur structure événementielle complexe. Des verbes comme casser, sécher (au sens de «faire sécher ») ou encore élargir sont par exemple causatifs, au sens où l'action du sujet induit une modification de l'argument objet. Leur structure événementielle correspond ainsi au schéma en (19a), et est instanciée respectivement tel qu'en (19b), (19c) et (19d), où x correspond à l'argument sujet et $y$ à l'argument objet :

(19) a. [[x agit] cause [y devient <état $>]]$

b. $[[\mathrm{x}$ agit $]$ cause $[\mathrm{y}$ devient $<$ cassé $>]]$

c. $[[\mathrm{x}$ agit $]$ cause $[\mathrm{y}$ devient $<$ séché> $]]$

d. [[x agit] cause [y devient <élargi> $]]$

Or selon Rappaport Hovav et Levin, à chaque sous-événement doit correspondre un argument spécifique, si bien que les verbes causatifs requièrent à la fois un sujet et un objet explicites. A l'inverse, des verbes comme frotter ou essuyer présentent une structure événementielle simple, non causative, et peuvent donc admettre l'omission de leur objet. 
L'analyse de Rappaport Hovav et Levin porte initialement sur l'anglais. Mais est-elle valable pour le français, et a fortiori pour le français parlé ? Une première difficulté pour évaluer cette analyse est bien sûr de faire le départ entre verbes causatifs et verbes noncausatifs, la distinction n'allant pas toujours de soi pour certains verbes. Mais si l'on garde à l'esprit le critère selon lequel un verbe est causatif si et seulement si il induit un changement dans l'argument objet, il apparait indéniable que les verbes effacer, casser, démolir, réparer, renouveler, compléter, couper, transformer, resserrer et polluer sont causatifs. Or tous ces verbes apparaissent dans le CFPP 2000 avec des objets implicites, invalidant ainsi l'approche lexicaliste événementielle, du moins pour le français :

(20) a. h. Bernard : c'est dingue on est dans une période où

Micheline : où on peut plus effacer

Sarah enq : $\mathrm{mm}$

Micheline : avant tu mettais les papiers à la cheminée + maintenant avec leurs

disques durs et tout ben excuse-moi mais +

Micheline : ils te coincent hein

Jean-Paul : oui oui + oui un + un un bon euh : un bon

Jean-Paul : informaticien il peut $: X X X$ là voilà

Sarah enq : il faut casser les disques durs [il suffit; si si] tu casses oui

Micheline : il peut il peut tu casses tu casses bien sûr tu casses

b. Christophe : euh + y avait + les bâtiments qui étaient sur le quai qui existaient + y avait encore la halle aux $v-+$ nous on a connu la halle aux vins encore donc les derniers les derniers récalcitrants quand même hein euh + la la majeure partie + était déja partie mais il restait quelques uns euh + autre XX c'était un chantier encore hein c'était vraiment

Marie-Anne : ils démolissaient

Christophe : ils démolissaient on a euh + on a fait nos études au moment de la démolition

c. donc aussi bien moi j'me souviens euh euh des orfè- des des bijoutiers qui faisaient un travail d'orfèvrerie y avait des matelassiers y avait des gens qui réparaient aussi donc des artisans d'art hein qu'on trouvait pour réparer euh j' me souviens c'était un type extraordinaire qui était euh rue + rue du Pré-aux-Clercs et qui a réparé toute une admirablement euh toutes sortes tout ce qui était porcelaine voilà objets cassés euh de de qualité + mais il réparait aussi bien entendu enfin vraiment et donc ils avaient euh toute une atmosphère

d. moi je suis un mauvais exemple parce que j'ai finalement à part euh + en tout en tout en tout huit ans d' ma vie donc où j'ai pas dû habiter là même pas sept ans d' ma vie où j'ai pas dû habiter là $(\mathrm{mh})++$ euh + ça change ça changerait complètement les habitudes mais après pourquoi pas de temps en temps faut bien faut bien renouveler e. et sinon vous n'êtes pas amenés à compléter encore en lisant je n' sais pas le Parisien ou euh + des journaux qui vraiment euh +

f. Reine : quoi + et oui puis moi j'ai trop Sarah enq : $j$ ' vois l' temps qui tourne terriblement et en même temps euh ++ c'est

Reine : oui mais enfin

Sarah enq : intéressant c'que vous

racontez donc j'ai pas envie d' couper

g. En parlant d'un magasin : 
comment ils ont rentab- enfin comment ils ont transformé tu vois +

h. oui oui + absolument + euh + je + je vous ai pas demandé alors + si je continue à

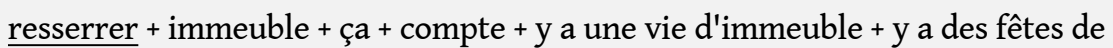
voisins?

i. des mauvaises fréquentations des gens de + venus de l'extérieur qui viennent traîner au Champ-de-Mars qui polluent et tout mais euh moi moi j'ai jamais eu d'incident dans la la dessus et le fait est que moi à chaque fois que j'vais au Champde-Mars + $y$ a toujours la police + qui tourne

Ainsi, un examen attentif du CFPP 2000, c'est-à-dire de données orales produites spontanément, dans une ignorance complète des enjeux linguistiques qui sous-tendent la démarche des enquêteurs, permet de montrer que les approches lexicalistes, qu'elles soient arbitraires, aspectuelles ou événementielles, sont inadaptées à rendre compte de la distribution des objets implicites en ce qui concerne le français parlé. Dans la section suivante, je propose une toute autre approche, de nature cette fois non plus lexicale mais pragmatique, fondée sur la notion de proéminence informationnelle.

\section{L'omission de l'objet réanalysée en terme de proéminence informationnelle du verbe sur l'objet}

Face aux approches lexicalistes de la distribution des objets implicites, dont j'ai montré dans la première section qu'elles ne parviennent pas à rendre compte de manière satisfaisante des données du français parlé, je propose dans cette seconde section une nouvelle approche pragmatique ou informationnelle du phénomène de l'omission de l'objet, articulée selon le principe suivant:

la proéminence phonologique du verbe sur l'objet sémantique (c'est-à-dire la réalisation phonologique du verbe et la non-réalisation de l'objet sémantique) traduit une proéminence d'ordre informationnelle: un objet sémantique est laissé implicite si et seulement si l'action décrite par le verbe est plus saillante que l'objet sémantique sur un plan informationnel.

A partir du corpus, je montre que trois cas se présentent. Tout d'abord, comme l'ont mis en évidence Lambrecht \& Lemoine (2005), lorsque un OID est pronominalisable, celui-ci correspond à une information topique, par opposition au verbe qui fait partie du commentaire : l'objet sémantique est ce dont on parle, l'information donnée, tandis que le verbe constitue le véritable apport informationnel concernant ce topique, d'où la proéminence informationnelle du verbe sur l'objet. Ce type d'objet implicite conforte ainsi l'hypothèse de Butt \& King (2000) selon laquelle un constituant nominal peut être laissé implicite si et seulement si il correspond à de l'information ancienne. En revanche, lorsque l'OID ne présente pas d'antécédent explicite et ne peut être pronominalisé, celuici ne correspond plus nécessairement à de l'information ancienne, contrairement à ce qu'affirment Butt \& King. Mais la thèse de Lambrecht \& Lemoine, selon laquelle l'OID ferait partie de l'information focalisée, semble également trop forte: comme nous le verrons, la notion d'information complétive mise en avant par Butt \& King semble plus adéquate pour décrire les OIDs non pronominalisables. En outre, Lambrecht \& Lemoine négligent également un autre type d'OIDs non-pronominalisables, qui quoique plus rare, apparait également dans notre corpus, à savoir les cas où un OID est anaphorique, mais présente comme antécédent un autre objet implicite : quoique non pronominalisable, ce 
type d'OIDs se rapproche alors du cas précédent des OIDs pronominalisables. Dans chacun de ces cas cependant, les données restent cohérentes avec notre hypothèse principale selon laquelle le verbe est informationnellement proéminent par rapport à l'objet implicite: en effet, qu'il s'agisse d'information topique ou d'information complétive, celles-ci restent informationnellement secondaires par rapport au focus. Enfin, dans le cas des OIIs, la proéminence informationnelle du verbe tient avant tout à ce que l'objet sémantique est de nature indéfinie : des indices peuvent éventuellement être inférés du contexte concernant la valeur sémantique de l'objet implicite, mais son identité précise n'importe pas, et c'est le verbe qui porte alors tout le poids informationnel.

\subsection{Les OIDs pronominalisables}

Lambrecht \& Lemoine (2005) distinguent deux types d'OIDs, parmi lesquels les OIDs qui présentent un antécédent explicite et sont ainsi pronominalisables. Selon eux, dans ce cas, l'OID correspond à un élément topique, c'est-à-dire à un référent identifiable et activé cognitivement à propos duquel le locuteur entend apporter de l'information pertinente. Le verbe quant à lui fait partie du commentaire ou focus, et constitue ainsi le véritable apport informationnel dans l'énoncé. Ce type d'OID illustre donc parfaitement l'hypothèse soutenue ici d'une proéminence informationnelle du verbe par rapport à l'objet implicite. Lorsque le verbe est énoncé, l'entité correspondant à l'objet implicite a déjà été activée, et ce sur quoi le locuteur veut attirer l'attention, ce qui constitue l'information nouvelle et pertinente, est bien l'information apportée sur le verbe. La proéminence phonologique du verbe par rapport à l'objet implicite est bien fonction de sa proéminence informationnelle.

Le corpus du CFPP 2000 illustre par un grand nombre d'exemples cette catégorie des OIDs pronominalisables, et met en outre en évidence que celle-ci recouvre en fait toute une gamme de cas particuliers qui s'articulent selon un continuum, plutôt qu'elle ne constitue une catégorie parfaitement homogène. En effet, un premier cas de figure concernant les OIDs pronominalisables correspond à la situation où l'OID est un véritable topique discursif. En d'autres termes, celui-ci est véritablement l'objet du discours, ou du moins d'une partie du discours, et donne lieu à de nombreux commentaires, dont la prédication correspondant au verbe à objet implicite. Ainsi, les verbes à objet implicite pointer et compter en (21a) s'inscrivent dans un ensemble de répliques portant sur le sujet des femmes voilées, les personnes interrogées s'étonnant de voir leur nombre augmenter à l'université; les femmes voilées, auxquelles correspondent les objets implicites des deux verbes, sont bien le topique discursif, donnant lieu à tout un ensemble de commentaires, dont la dernière réplique du passage mettant en jeu les deux verbes à objet implicite. De même, en (21b), les deux occurrences à objet implicite du verbe arrêter prennent place à la fin d'une discussion sur l'équitation, l'enquêteuse Florence ayant soulevé le débat des activités à Paris, et la jeune femme Lucie ayant rebondi sur sa pratique de l'équitation. Enfin, le passage en (21c) intervient au sein d'une longue évocation d'un lieu prénommé le Relax bar, auquel correspond l'OID de nettoyer; à nouveau, l'objet implicite correspond donc au topique discursif, sur lequel le verbe sans objet apparent vient donner une information nouvelle :

(21) a. Christophe : ah tout à fait + tout à fait + euh ce qui nous su- ce qui nous surprend plus euh + qui nous choque même c'est de de voir de plus en plus de jeunes femmes voilées venir à la fac ou des choses comme ça ça ça oui 
Enq Sarah : ça vous semble + ça s'est beaucoup accru récemment?

Christophe : oh oui je pense + je pense hein + même même depuis qu'on est là

Marie-Anne : oui moi j'ai l'impression mais enfin bon

Christophe : c'est + c'est très difficile à dire vous savez c'est des + c'est des

Christophe : sensations c'est des

Enq Sarah : c'est une impression comme ça et vous ne passez pas votre temps à

pointer et à compter

b. [à propos de l'équitation]

Florence Enq : ouais + là vous avez arrêté ?

Lucie : Euh là j'ai arrêté oui +

c. Bernard : mais le lieu existe toujours apparemment il semble fermé

Micheline : mais il est fermé + voilà

Sarah enq : et : : la population

Jean-Paul : euh oui oui c'est fermé ils ont net- ils ont nettoyé

L'OID correspond parfois également à un topique discursif qui s'étend simplement sur quelques répliques, l'enquêteuse soulevant un sujet sous la forme d'un topique de phrase, et les personnes interrogées commentant alors ce topique à l'aide d'un verbe à objet implicite :

(22) a. SBenq : les cours d'ELCO vous avez suivi

Youcef : non moi j'ai pas suivi

b. SBenq : l'argot des Puces vous l'aviez appris ?+

Youcef : ah oui ça

Abdel : j-je ben oui je je

Youcef : oui + c'est sûr

Abdel : j'ai appris mais je j'le parlais jamais + j'aimais pas

Ces différents exemples d'OIDs correspondant à un topique discursif illustrent clairement cette idée d'une proéminence informationnelle du verbe sur l'objet implicite, ce dernier correspondant à une entité déjà soulevée par le discours, à propos de laquelle le verbe vient apporter de l'information nouvelle.

Mais les OIDs pronominalisables peuvent aussi simplement correspondre à un topique de phrase. Dans ce cas, l'entité correspondant à l'OID a été soulevée précédemment, mais sous la forme d'une information focus, c'est-à-dire d'une information nouvelle. Et ce n'est que dans la proposition contenant le verbe à objet implicite que cette entité acquiert le statut de topique. En d'autres termes, c'est le verbe lui-même qui, en prenant cette entité pour objet implicite, la fait accéder au statut de topique. En témoignent notamment les exemples suivants :

(23) a. on sortait les écrits militaires de Marx et d' d'Engels on sortait les écrits mieuh Trotsky et alors j'allais en leur disant "mais achetez au moins"

b. donc vous avez parlé + des + changements intervenus dans les commerces + vous pouvez préciser?

c. Reine : elle elle fait des très bons gâteaux d'ailleurs il nous reste un tout petit morceau + (rire)et ça

Reine : tu vas goûter

d. oui tous les jours on nous rappelle qu'y a l' bac tous les jours on nous rappelle que 
$(\mathrm{mh})++$ donc euh $+\mathrm{c}^{\prime}$ est un peu oui c'est une pression y en a qui supportent pas à mon avis

e. et puis euh + y avait une bonne offre qui s'est proposée à moi donc j'ai saisi et puis $(\mathrm{mm} \mathrm{mm}) \mathrm{c}^{\prime}$ est dans un quartier que je connais déjà donc + voilà l'introduction, du fait de la difficulté à établir s'il mettent en jeu des constituants disloqués, c'est-à-dire détachés en périphérie, ou bien de vraies expressions syntaxiquement topicalisées, c'est-à-dire déplacées de leur position de complément d'objet direct :

(5) a. tu vois l' photographe à côté je fais travailler bon la presse elle est à côté b. mais c'est vrai que cinquième sixième septième j'connais bien et j'aime beaucoup c. euh alors la Pyramide $+j$ 'suis pas $+j$ 'ai jamais vu avant en fait d. le Carrousel les lumières euh + j'aime beaucoup

e. le quartier populaire euh + du donc du nord du de l'est + + vous connaissez un petit peu

f. l'automobiliste on taxe déjà beaucoup

S'il s'agit de constituants disloqués, alors il est indéniable que ces énoncés mettent en jeu de véritables objets implicites, et non des compléments d'objet directs qui ont simplement été déplacés; ces constituants disloqués illustrent ainsi le cas évoqué cidessus où l'entité correspondant à l'objet implicite et jouant dans la phrase un rôle de topique informationnel est explicitement représentée. Mais une question qui peut être soulevée est de savoir si même les énoncés mettant en jeu un véritable topique syntaxique, tels que le complément d'objet a été déplacé de sa position de complément d'objet vers une position de complémenteur, ne peuvent pas dans une certaine mesure également être subsumés sous le schéma général décrit dans cette section. En effet, même si le complément d'objet est déplacé, il n'en reste pas moins que l'espace correspondant à sa position initiale est lui-même interprété, et qu'il coréfère avec un élément topique, ici un topique phrastique exprimé sous une forme explicite. Il s'agit là d'une hypothèse qui 
mériterait assurément une réflexion plus approfondie, dépassant le cadre restreint de cet article.

Topique discursif ou topique phrastique, topique implicite ou bien explicité, les différents cas examinés ci-dessus confirment donc bien la thèse de Lambrecht \& Lemoine (2005) selon laquelle les OIDs pronominalisables correspondent à un élément topique. Cependant, le CFPP 2000 permet, non pas de contredire cette affirmation, mais de la nuancer, en montrant que les OIDs pronominalisables correspondent en effet toujours à des entités qui ont déjà été activées dans le discours (ou bien, cas qui ne se présente pas dans notre corpus, par la situation extra-discursive), mais dont la saillance topicale peut être plus ou moins forte. De fait, certaines occurrences d'OIDs pronominalisables dans notre corpus correspondent à un élément dont on ne peut dire qu'il soit le topique de la phrase, et encore moins le topique discursif, mais qu'il constitue bien plutôt un élément topique parmi d'autres, qui n'a pas été activé plus fortement que les autres éléments dans le contexte précédent. En (25a) par exemple, l'OID d'occuper est paraphrasable successivement par le pronom vous puis par le pronom nous, c'est-à-dire qu'il correspond aux jeunes filles Katia et Julie interrogées par l'enquêteuse. Cependant, à ce moment du discours, ces individus n'ont pas été activés comme référent de discours plus fortement que d'autres entités et ne peuvent donc revendiquer le rôle de topique. En témoigne d'ailleurs la présence du topique explicite ces discussions politiques au début du passage, qui semble en fait correspondre à l'élément topique principal, suscitant les différents commentaires qui vont suivre. De même, en (25b) et (25c), les objets implicites de pousser et de marquer sont tous deux paraphrasables par le pronom nous, mais l'entité à laquelle celui-ci correspond n'a pas été activée plus fortement que les autres entités de discours, et si véritable et unique topique phrastique il y a, celui-ci correspond plutôt aux films comme ça évoqués en début d'énoncé en (25b), et au taxidermiste dont le magasin a brûlé en $(25 c)$ :

(25) a. Sonia B.-R. Enq : ces discussions politiques elles + elles occupent + un petit moment

Katia Teixeira : oui + oui non ++

Sonia B.-R. Enq : ou elles sont importantes

Katia Teixeira : non elles occupent un petit moment mais euh enfin c'est surtout

l'avis des parents qui est répercutant sur la vie des enfants enfin

b. des des films comme ça mais c'est pas : : c'est pas mon père qui poussait

c. oui + le taxidermiste et c'est vrai que ça a ++ dans l' quartier on en a beaucoup parlé parce que c'est c'est quand même + pour les enfants d'abord c'est XX $(\mathrm{mh})$ mais aussi pour les parents c'est un + c'était un lieu un peu mythique quoi où + où on aimait euh aller d' temps à autres (oui) où ++ euh ça ça a beaucoup marqué

53 Ainsi, le corpus montre que les OIDs pronominalisables peuvent revêtir le statut de topique informationnel à des degrés et à des niveaux divers, pouvant être topiques de phrase ou topiques de discours, et correspondant tantôt à l'élément le plus fortement topicalisé, tantôt à un élément topique parmi d'autres.

Cependant, quelle que soit la spécificité des différents cas évoqués dans cette soussection, tous concordent avec notre hypothèse principale selon laquelle un verbe n'admet un objet implicite que s'il est informationnellement proéminent par rapport à lui. En outre, ils confortent également l'hypothèse de Butt \& King (2000), qui affirment que seul 
un groupe nominal correspondant à de l'information ancienne ou connue peut être laissé implicite. De fait, quel que soit son degré de topicalité, un élément topique correspond nécessairement à une entité déjà activée dans le discours. Mais ces deux hypothèses sontelles également confirmées par le second type d'OID répertorié, à savoir les OIDs non pronominalisables?

\subsection{Les OIDs non pronominalisables}

Comme souligné par Lambrecht \& Lemoine (2005), les OIDs non pronominalisables sont des OIDs qui ne présentent pas d'antécédent explicite, discursif ou extra-discursif, et ne correspondent donc pas à une entité déjà activée cognitivement. Leur valeur est entièrement inférée à partir du contexte et de la valeur sémantique du verbe. De fait, Lambrecht \& Lemoine décrivent les OIDs non pronominalisables comme des OIDs "induits par un frame», au sens Fillmorien où un prédicat, et notamment un verbe, induit un scénario impliquant différents participants. On peut trouver dans le CFPP 2000 un grand nombre d'exemples de ce type d'OIDs (l'OID est ici explicité par l'expression entre crochets pour des raisons de lisibilité, celui-ci étant souvent difficile à identifier sans un contexte plus étoffé) :

(26) a. alors on va reprendre [la conversation]

b. oui oui + absolument + euh + je + je vous ai pas demandé alors + si je continue à resserrer [le thème de la conversation] + immeuble + ça + compte + $y$ a une vie d'immeuble + $\mathrm{y}$ a des fêtes de voisins?

c. Reine : quoi + et oui puis moi j'ai trop

Sarah enq : $\mathrm{j}^{\prime}$ vois l' temps qui tourne terriblement et en même temps euh + + c'est Reine : oui mais enfin

Sarah enq : intéressant c'que vous racontez donc j'ai pas envie d' couper [le magnétophone]

d. l'promoteur immobilier qui a fermé [son agence immobilière] aussi mais il avait ouvert [son agence] six mois avant donc euh + ça sent l'détournement d'argent e. on + peut dire les choses + généralement comment vous faites pour l'entretien de la maison + pour euh vous partagez [les tâches] + ou vous trouvez chacun XX f. dans Paris euh un petit peu quand même parce que y a eu euh des arrivées quand même de qui se sont multipliées euh de cuisines donc on est allés chez des oui c'est vrai des Japonais puis on s'est un peu lassés des sushis + alors on on varie [les restaurants que l'on choisit] quoi

g. qu'est-ce qui se passe quand on a un enfant d'é- + étranger est-ce qu'il rattrape [le niveau] euh

h. et sinon vous n'êtes pas amenés à compléter [vos lectures] encore en lisant je n' sais pas le Parisien ou euh + des journaux qui vraiment euh +

i. + il nous traitait des fois de tous les noms parce qu'on avait pas fait c' qu'il vous fallait + mais c'était un docteur comment dire de famille un docteur + comme un docteur de campagne comme il demandait jamais d'argent si on pouvait pas payer [la consultation] il disait rien

j. Lies : mais est-ce que c'est parce que tu tu penses ne pas avoir les moyens ou est-c' que c'est par prudence ?+

Isabelle : ben j'pense que c'est un peu de c'est un peu des deux enfin c'est plus par prudence en $\mathrm{m}$ ' disant euh + "j' vais limiter [mes dépenses] parce qu'on 
Isabelle : sait jamais"

Lies : sait oui voilà

k. non + je pense que quand même dans l' quartier + euh + il faut reconnaître une chose c'est que + les maîtres ramassent [les crottes de leur chien]

Dans chaque cas, l'objet implicite du verbe doit être précisément identifié pour que la communication s'effectue avec succès, et cette valeur sémantique définie ne peut être identifée sur la seule base du contenu lexical du verbe, même si celle-ci constitue également une source d'information importante. Ainsi, pour ne prendre qu'un exemple, l'objet implicite de resserrer en (26b) pourrait être tout autre dans un contexte différent, et c'est grâce au contexte spécifique dans lequel il apparaît que l'on peut comprendre que l'OID correspond au thème de la conversation en cours.

57 Mais quel est au juste le statut informationnel spécifique de ce type d'OIDs? Selon Lambrecht \& Lemoine (2005), explicités, ces éléments appartiennent au focus. Mais est-ce à dire que sous leur forme implicite, ils font également partie de l'information focus ? Sur un plan informationnel, si l'on définit le focus comme l'information nouvelle venant commenter dans un énoncé un élément topique, déjà connu, cette hypothèse apparaît plausible, puisque les OIDs pronominalisables ne correspondent pas à une entité déjà activée cognitivement. Cependant, un focus informationnel peut être éventuellement aussi un focus prosodique; or par définition un élément implicite ne peut être phonologiquement focalisé. En outre, même s'ils correspondent tous deux à de l'information nouvelle, il semble difficile de réduire le verbe et l'OID non pronominalisable à un seul et même type d'information; car tandis que le verbe apporte une information véritablement nouvelle, qui n'est pas identifiable sur la base du seul contexte, l'OID pronominalisable quant à lui est entièrement inféré à partir du contexte précédent, c'est-à-dire à partir de la valeur lexicale du verbe et du contexte élargi. Ainsi, l'hypothèse de Butt \& King selon laquelle un constituant implicite correspond nécessairement à de l'information connue apparaît mise à mal par le cas des OIDs nonpronominalisables. Néanmoins, la typologie de Butt \& King, qui distinguent deux sortes d'information nouvelle, peut en fait nous aider à identifier de manière plus satisfaisante le statut informationnel de ce type d'OIDs. Selon elles, l'information nouvelle se divise entre information focus et information complétive : contrairement à l'information focus, l'information complétive n'est pas phonologiquement focalisée, et elle est "nouvelle, mais non proéminente ${ }^{5} »$. Selon cette typologie, le verbe correspond donc bien à de l'information focus, tandis que l'OID non pronominalisable serait de l'information complétive. Et si tel est le cas, bien que constituant de l'information nouvelle, l'OID non pronominalisable est informationnellement en retrait par rapport au verbe, conformément à notre hypothèse selon laquelle le verbe occupe nécessairement un statut proéminent par rapport à son objet implicite sur un plan informationnel.

Le corpus CFPP 2000 permet cependant de montrer une exception à cette analyse des OID non pronominalisables, en mettant en évidence une sous-catégorie d'OIDs non pronominalisables qui sont en fait plus proches du comportement des OIDs pronominalisables que du comportement de la majorité des OIDs non pronominalisables tels que décrits ci-dessus. De fait, le corpus met en évidence des cas où un OID présente un antécédent, mais un antécédent correspondant lui aussi à un objet implicite, comme en (27) : 
(27) a. mais moi j'ai mon père il voulait toujours m' donner mais moi j'aimais [event : noise next $\mathrm{mm}$ ] pas trop prendre +

b. il écrit comme il prononce + c- comme il entend +

c. euh y a pas mal de $+\mathrm{de}+$ parents qui achètent pour leurs enfants étudiants + et qui revendent après +

En (27a), l'objet implicite de donner est paraphrasable par de l'argent, et cette entité est ensuite reprise sous une forme implicite définie comme objet implicite de prendre. En (27b), l'énoncé signifie que le sujet écrit une chose comme il la prononce et l'entend: l'antécédent de l'OID de prononcer et entendre est donc bien l'objet implicite d'écrire. Enfin, en (27c), acheter prend tout d'abord un OII que l'on identifie facilement à un appartement, et c'est cet élément qui sert ensuite d'antécédent à l'OID du verbe revendre. Ces OIDs, bien que non pronominalisables, diffèrent ainsi de la majorité des OIDs non pronominalisables en ce qu'ils ne constituent pas de l'information nouvelle, et se rapprochent des OIDs pronominalisables en ce qu'ils prennent un antécédent discursif. La différence avec ces derniers est que cet antécédent est lui-même implicite, empêchant ainsi la pronominalisation de l'OID. Mais de même que l'on se refusait à accorder le même statut informationnel aux OIDs non pronominalisables évoqués précédemment et au verbe, à savoir le statut d'information focus, de même, peut-on accorder à ce nouveau type d'OIDs non pronominalisables le statut d'information topique, précédemment accordé aux OIDs pronominalisables? L'impossibilité de pronominaliser un OID recevant comme antécédent un autre objet implicite suggère que cet amalgame serait réducteur, et que ce second type d'OID pronominalisable ne peut prétendre comme les OIDs pronominalisables au statut d'entité cognitivement activée, ou du moins pas à un même degré que ne peuvent l'être les OIDs pronominalisables. A nouveau, la typologie de Butt \& King peut s'avérer alors utile, dans la mesure où elle distingue au sein de l'information ancienne d'une part l'information topique et d'autre part l'information d'arrière-plan, la seconde étant informationnellement seconde par rapport à la première. Tandis que les OIDs pronominalisables correspondent indéniablement à de l'information topique, à propos de laquelle est effectué le commentaire correspondant au verbe, l'antécédent implicite d'un OID reste lui une entité informationnellement secondaire, qui n'est pas activée au même point qu'une véritable information topique et ne permet donc pas la pronominalisation de l'OID : elle constitue ainsi une information d'arrière-plan, et non une information topique.

60 Comme pour les OIDs pronominalisables, les données du CFPP 2000 permettent ainsi de découvrir une diversité de comportements pour les OIDs non pronominalisables, ces derniers correspondant tantôt à de l'information complétive, tantôt à de l'information d'arrière-plan, selon la typologie de Butt \& King. Néanmoins, dans la mesure où information complétive et information d'arrière-plan correspondent toutes deux à un type d'information moins saillant que l'information focus, chacun des cas étudiés vient confirmer la thèse principale de cet article, à savoir l'idée que la proéminence phonologique d'un verbe sur son objet implicite s'explique par une proéminence d'ordre informationnel. 


\subsection{Le cas des Olls}

61 Enfin, les OIIs achèvent de confirmer la thèse d'une proéminence informationnelle du verbe sur l'objet implicite, quoique sur un autre mode que les OIDs examinés précédemment. Dans le cas des OIIs, la proéminence informationnelle du verbe tient à ce que l'objet sémantique est de nature indéfinie : son identité précise ne constitue pas une information pertinente, et c'est l'action exprimée par le verbe qui reçoit toute l'attention. Dans le cas standard, la valeur sémantique de l'OII ne reçoit pour seule contrainte que les restrictions sélectionnelles imposées par le verbe lui-même. Ainsi, en (28), l'OII de manger est interprété comme une/des chose(s) mangeable(s), celui de cuisiner correspond à des plats, celui de composer à de la musique, celui de lire à des choses lisibles et ainsi de suite... A chaque fois, la valeur sémantique de l'oII est entièrement déterminée par le contenu lexical du verbe et reste indépendante du contexte :

(28) a.on peut trouver des restos où on mange pour quinze euh pour quinze euros à deux

b. euh je j'X même globalement j'cuisine plus finalement euh au mois d'septembre

c. moi j'écris d'la musique donc j'compose et euh et ça j'le fais chez moi la plupart du temps

d. c'est un avantage on va dire plutôt que d'aller s'déplacer : : pour aller prendre le des bains d'soleil ou pour aller lire dans un parc + on est juste à côté +

e. donc les gens savent pas coudre + du tout + et donc euh faire un ourlet c'est un problème

f. aujourd'hui on a plus d'référence on est on est habitué à à l'euro et on (n') achète plus euh d'la même manière aujourd'hui on dit on s'dit qu'un euro c'est pas cher mais avant un franc c'était pas cher un euro c'est cinq cinq six francs donc ça a rien à voir euh c'est

g. on sortira moins ou on consommera moins... à part euh un moment exceptionnel peut-être mais on consommera peut-être un petit peu moins on fera peut-être un peu plus attention

i. juste le fait d'apprendre à multiplier ça m'a ça m'a fait adorer les mathématiques vraiment

62 Ainsi, l'objet implicite étant réduit à sa plus simple valeur sémantique, celle qui résulte de l'application des restrictions sélectionnelles du prédicat verbal, c'est bien le verbe luimême qui porte toute l'information. Pour ne prendre qu'un exemple, en (28i), l'apport informationnel provient tout entier de l'action décrite par multiplier; du fait de notre compréhension du lexique, nous savons que l'objet implicite de ce verbe ne peut correspondre qu'à des nombres, mais leur identité, leur valeur précise n'importe pas ici. D'un point de vue informationnel, le verbe est très nettement proéminent par rapport à son objet sémantique.

63 Mais les OIIs peuvent également recevoir des restrictions sémantiques plus fortes que celles uniquement imposées par le verbe, et leur valeur sémantique peut être restreinte par le contexte. Ainsi, si l'on considère par exemple acheter en (29a), son OII n'est pas interprété seulement comme des choses achetables mais comme de la nourriture, car la première partie de l'énoncé focalise l'attention sur la question du prix de la nourriture. De même en (29b), l'OII de consommer est restreint à des produits bio car la discussion 
porte spécifiquement sur l'achat de produits dans des marchés bio, tandis qu'en (29c), la valeur restreinte de l'OII, à savoir une voiture, a déjà été explicitée dans la réplique précédant l'énoncé contenant le verbe à objet implicite louer. En (29d) et (29e), l'OII de acheter et celui de chercher sont tous deux paraphrasables par un appartement, puisque la discussion porte respectivement sur le fait d'emménager et sur celui de déménager. En (29f), même sans être accoutumé à un tel usage de fréquenter, on comprend que l'OII du verbe correspond à des garçons, tandis qu'en (29g), il ne s'agit pas de retrouver une simple chose ou une autre, mais bien un travail. En (29h), ce sont les propos de Jacqueline Pelletier qui permettent de comprendre que l'OII de ramasser et celui de chercher sont approximativement paraphrasables par des choses dans les poubelles. Enfin, en (29i), c'est le contexte du café qui permet de comprendre que l'OII de reprendre correspond à une boisson:

(29) a. moi j'vois les prix qui augmentent mais bon faut bien manger donc j'achète hein enfin euh

b. Benoît : et le vendredi euh on a un marché bio alors on y va

Enq SB : marché bio aussi

Benoît : oui mais on consomme pas trop hein parce que l'bio c'est trop cher

c. Christophe : c'est assez rare + mais enfin oui on loue les voitures euh + par exemple on a des amis qui habitent en banlieue euh + en grande banlieue hein

Enq Sarah : oui alors justement euh + que +

Christophe : on on loue une voiture

Enq Sarah : ah là vous louez

d. donc euh : c'est vrai qu'c'est ça qu'à mon avis qu'a fait que l'quartier est très catrès très ca(lme) très très très calme à l'époque + et en fait on voit de plus en plus de jeune qu'arrivent vers ce quartier là qui emménagent qui achètent euh pour euh : des jeunes mariés avec leurs leurs enfants

e. nous ha- nous avions acheté un petit appartement au sixième sans ascenseur dans le huitième arrondissement et nous cherchions à nous agrandir nous avons cherché partout

f. elles fréquentaient bien avant moi : euh bien plus jeunes que moi et : $:+$ bien plus assidûment hein elles variaient leurs copains tandis que moi euh + pas trop [rires] g. j'avais travaillé mais quand j'me suis mariée + j'avais cessé complètement mon travail donc avant de retrouver dans l'secrétariat $(\mathrm{mm}) \mathrm{j}$ 'ai fait différents + h. Jacqueline Pelletier : et ben là j'vous assure que vous y en a plein des gens très très pauvres $(\mathrm{mm})$ moi j'vois des + hier encore $(\mathrm{mm})$ j'ai été choquée $(\mathrm{mm})$ j'ai été voir mes copines j'ai été $m$ 'faire faire une prise de sang + donc j'ai été à l'Action sociale euh $(\mathrm{mm})$ à Pablo $(\mathrm{mm})$ donc c'était vraiment ils remballaient + les + au marché $(\mathrm{mm})$ ben si vous saviez c'que j'ai vu

Enq SBR : le nombre de gens qui qui ramassent Jacqueline Pelletier :j'ai vu comme + de personnes âgées Jacqueline Pelletier : qui étaient là qui cherchaient + ben vous savez quand vous voyez ça ça m'fous les boules hein

i. de de le les gens qui tiennent ça c'est des Kabyles oui $(\mathrm{mm})$ + mais enfin les il reçoit tout l'monde hein y a pas qu' des Kabyles y a même des Roumains qui habitent dedans + $(\mathrm{mm})+$ c'est un café où on v- on n'essaie pas de vous tirer d' l'argent quoi y a des cafés où $\mathrm{X}$ on essaie de vous tirer $\mathrm{d}$ ' l'argent + + là vous allez vous prenez un 
café personne vous demande "il faut reprendre" ou vous restez vous discutez y a des gens qui jouent aux dominos ce que requiert la valeur lexicale du verbe, une valeur fortement modulée par le contexte discursif, quoique toujours indéfinie : seul le contenu prédicatif de l'OII se trouve en fait modifié par le contexte, non son indéfinitude. Or le point crucial de cette démonstration est que ces OIIs, même avec une sémantique plus restreinte, demeurent informationnellement en retrait par rapport au verbe. Pour le dire autrement, même si le contexte permet d'identifier avec plus de précision dans quel domaine les entités correspondant à l'objet sémantique peuvent être trouvées, leur identité précise reste non pertinente dans le cadre de la discussion, et c'est l'action décrite par le verbe qui demeure l'élément informationnellement le plus proéminent. Pour faire un parallèle avec les OIDs non pronominalisables (ceux qui ne présentent aucun antécédent, pas même un antécédent implicite), l'on peut faire l'hypothèse que les OIIs correspondent également à de l'information complétive, selon la typologie de Butt \& King (2000) : ils constituent bien une information nouvelle, mais ils ne peuvent être prosodiquement focalisés, et leur apport informationnel est nettement en deçà de celui du verbe, qui, lui, apporte l'information première et exprime l'information focale. Ainsi, de même que les autres objets implicites examinés ici, les OIIs apparaissent informationnellement moins proéminents que le verbe.

\section{Conclusion}

Pour conclure, l'originalité de cet article se manifeste essentiellement par deux apports spécifiques. D'une part, ce travail sur corpus démontre que les hypothèses lexicalistes majeures qui ont été avancées dans la littérature concernant la distribution des objets implicites ne permettent pas en elles-mêmes d'expliquer le phénomène dans le français parlé. D'autre part, cet article propose une approche informationnelle de la distribution des objets implicites fondée sur une hypothèse unifiante, qui permet de rendre compte des différents types d'objets implicites que constituent les OIDs pronominalisables, les OIDs non pronominalisables et les OIIs, tout en préservant la spécificité de chaque catégorie, voire les particularités intra-catégorielles. Dans quelle mesure cette analyse s'applique-t-elle spécifiquement au français parlé? Dans quelle mesure l'omission de l'objet dans le français écrit diffère-t-elle du phénomène tel qu'observé dans le français parlé ? Une hypothèse possible est que les contraintes lexicales pesant sur l'omission de l'objet, bien qu'apparemment nettement surmontées par des enjeux d'ordre informationnel à l'oral, soient en fait réelles et ressortent de manière plus marquée dans le français écrit. Ce modèle mixte entre contraintes lexicales et contraintes informationnelles dont la part respective varierait selon qu'il s'agit du français écrit ou du français oral apparaît d'autant plus plausible que l'oral est de manière générale le lieu privilégié d'une prise de liberté par rapport aux contraintes grammaticales et notamment aux contraintes lexicales, non pas pour céder le pas à l'anarchie linguistique mais pour donner la part belle à des enjeux d'ordre pragmatique, communicationnel, ou en d'autres termes informationnel. Cette question d'une comparaison de la part respective des contraintes lexicales et des contraintes informationnelles dans le phénomène de

TIPA. Travaux interdisciplinaires sur la parole et le langage, 30 | 2014 
l'omission de l'objet à l'écrit d'une part et à l'oral d'autre part constitue un enjeu passionnant, auquel ce travail ouvre la voie.

\section{BIBLIOGRAPHIE}

Butt, M. \& T. H. King, T. H. (2000) Null elements in discourse structure, in Subarao, K. V. (ed), Papers from the NULLS Seminar. Delhi : Motilal Banarsidass.

Branca-Rosoff, S., Fleury, S., Lefeuvre, F. \& M. Pires (2009) Discours sur la ville. Corpus de Français Parlé Parisien des années 2000 (CFPP2000) http://cfpp2000.univ-paris3.fr/

Branca-Rosoff, S., Fleury, S., Lefeuvre, F. \& M. Pires (2012) Discours sur la ville. Présentation du Corpus de Français Parlé Parisien des années 2000 (CFPP2000) http://cfpp2000.univ-paris3.fr/ CFPP2000.pdf

Fillmore, C. J. (1986) Pragmatically Controlled Zero Anaphora, in Nikiforidou, V., Vanllay, N., Niepokuj, M. \& D. Felder (eds.), Proceedings of the Twelfth Annual Meeting of the Berkeley Linguistics Society, Berkeley, CA: BLS, p. 95-107.

Gillon, B. (2012) Implicit complements: a dilemma for model theoretic semantics, Linguistics and Philosophy, 35, p. 313-359.

Lambrecht, K. \& K. Lemoine (2005) Definite null objects in (spoken) French. A constructiongrammar account, in Fried M. \& H. Boas (eds.), Proceedings of the First International ConstructionGrammar Conference, Amsterdam, Philadelphia : Benjamins, p. 13-55.

Martí, L. (2009) Grammar vs. Pragmatics: Carving Nature at the Joints, ms., University of Tromsø. Mittwoch, A. (1971) Idioms and Unspecified NP Deletion, Linguistic Inquiry 2, p. 255-259.

Mittwoch, A. (1982). On the Difference between Eating and Eating Something: Activities versus Accomplishments, Linguistic Inquiry, vol. 13, 1, p. 113-122.

Noailly, M. (1994) Adjectif adverbal et transitivité, Cahiers de grammaire, 19, p. 103-114.

Noailly, M. (1997) Les mystères de la transitivité invisible, in Langages, 127, p. 96-109.

Noailly, M. (1998), Transitivité absolue et type de prédication, in M. Forsgren, K. Jonasson \& H. Kronning (eds.), Prédication, assertion, information: Actes du colloque d'Uppsala en linguistique française, 6-9 juin 1996, Uppsala : Acta Universitatis Uplsaliensis, p. 377-384.

Rappaport Hovav, M. \& B. Levin (1998) Building Verb Meanings, in M. Butt \& W. Geuder (eds.), The Projection of Arguments: Lexical and Compositional Factors, CSLI Publications, Stanford, CA, p. 97-134.

\section{NOTES}

1. Je traduis de l'anglais.

2. Pour tout exemple tiré du corpus, je souligne le verbe à objet implicite faisant l'objet de la démonstration. 
3. Pour le sens des signes diacritiques, voir Branca-Rosoff et al. (2012).

4. Comme le souligne un rapporteur, hors contexte, le dernier exemple (18e) est ambigu entre une première interprétation où l'enquêteuse demande si les protagonistes ont assisté aux cours d'ELCO, et une seconde interpretation où elle leur demande s'ils les ont compris. Dans ce second cas, on pourrait alors considérer que l'OID de suivre ne correspond pas précisément aux cours d'ELCO mais est plutôt paraphrasable par le contenu des cours d'ELCO. Le contexte permet cependant de désambiguïser le propos en faveur de la première interpretation, où suivre est employé comme synonyme d'assister à, et telle que l'OID est bien paraphrasable par les cours d'ELCO.

5. Le terme original en anglais est prominent.

\section{RÉSUMÉS}

Cet article consiste en une étude du phénomène de l'omission du complément d'objet dans le français parlé, réalisée à partir du Corpus de Français Parlé Parisien des années 2000 (CFPP 2000). Plusieurs approches de type lexicaliste ont été défendues dans la littérature, selon lesquelles la capacité d'un verbe transitif à admettre ou non les objets implicites est déterminée à un niveau lexical. En effet, cette propriété est considérée tantôt comme étant fixée de manière arbitraire dans le lexique, tantôt comme résultant des caractéristiques aspectuelles du verbe, ou encore comme résultant de ses caractéristiques événementielles. Dans cet article, à partir du CFPP 2000, je montre tout d'abord qu'aucune des approches lexicalistes ne parvient à rendre compte de manière satisfaisante du phénomène de l'omission de l'objet tel qu'il se réalise dans le français parlé. Puis, dans une seconde section, je propose une analyse alternative, de nature non plus lexicaliste mais pragmatique, selon laquelle la proéminence phonologique du verbe sur son objet implicite est fonction de sa proéminence informationnelle. Je montre que cette analyse parvient à rendre compte des différents types d'objets implicites que constituent les objets implicites définis pronominalisables, les objets implicites définis non pronominalisables, et enfin les objets implicites indéfinis.

An element $\mathrm{X}$ is said to be more prominent than an element $\mathrm{Y}$ whenever $\mathrm{W}$ receives more emphasis, and is more salient than $Y$. On a phonological level, the highest degree of prominence of a linguistic item $\mathrm{X}$ compared to another linguistic item $\mathrm{Y}$ is the case where $\mathrm{X}$ is phonologically realized and $\mathrm{Y}$ is not, as illustrated for example by the phenomenon of verbs with implicit objects. Indeed, some verbs are semantically transitive and hence necessarily require a semantic object, but can nevertheless appear without a direct object that is phonologically realized : the semantic object is then implicit. Thus, a verb like manger 'eat' for example can be used without an explicit direct object, as in (1) :

1. Jean est en train de manger.

'John is eating'

The utterance is most naturally understood as meaning that John is eating something, a thing whose precise identity does not matter : manger is here interpreted with an implicit indefinite object (or IIO). But if I utter (2) while throwing a ball to someone,

2. Attrape!

'Catch (it)'

my utterance will then be understood as an incentive to catch not something or other, but 
precisely the ball I am throwing : attrape 'catch' is then interpreted with a definite implicit object (or DIO). However, other verbs prima facie seem to have more difficulties admitting implicit objects. Thus, the utterances in (3) and (4) for example do not sound fully acceptable; the object omission here appears quite difficult with the verb faire 'make' :

3. Marie est en train de faire

'Mary is making'

4. Fais!

'Make!'

Which factors can explain the acceptability of the omission of the object complement, and the definite or indefinite interpretation of the implicit object?

Several approaches of a lexicalist kind have been defended in the literature - among which many for the English language - on which the ability of a verb to admit IIOs or DIOs corresponds to a lexical property of the verb. Thus, Fillmore (1986) first and then more recently Gillon (2012) have contended that this corresponds to a property that is determined within the lexicon in a fully arbitrary way, and cannot be inferred from other semantic properties of the verb. Mittwoch $(1971,1982)$ and Noailly (1998) prefer to analyze the ability of admitting IIOs or DIOs as the result of aspectual properties of the verb ; thus, verbs admitting IIOs would be atelic verbs, while verbs admitting DIOs would be telic. Finally, Rappaport Hovav and Levin (1998) develop a so-called 'event structure' approach, according to which causative verbs do not admit object omission due to their complex event structure. Yet, an obvious limit of the lexicalist approach is that such analyses are often based on written corpora, or even on invented examples, that might thus be possibly biased, the latter because they rely on introspection, the former because they reflect a very constrained use of the language, that can only imperfectly illustrate the phenomenon as it is realized in spoken French.

In this paper, I rely on a corpus of Parisian Spoken French from the 2000s, the CFPP 2000, to show that lexicalist approaches are unable to account for the phenomenon of object omission as it is realized in spoken French. I argue that object omission is guided by communicational criteria, according to the following principle :

The phonological prominence of the verb on the semantic object (that is, the phonological realization of the verb and non-realization of the semantic object) reflects an informational prominence : a semantic object is left implicit if and only if the action described by the verb is more salient than the semantic object on an informational level.

Based on the corpus, I distinguish three cases. First, as has been put forward by Lambrecht \& Lemoine (2005), whenever a DIO is pronominalizable, it corresponds to a topical information, by contrast with the verb that is part of the comment : the semantic object is what is talked about, the given information, whereas the verb brings the genuine informational import about the topic, hence the informational prominence of the verb on the object. This kind of implicit object thus supports Butt \& King's (2000) hypothesis, according to which a nominal constituent can be left implicit if and only if it corresponds to old information. By contrast, whenever the DIO does not receive an explicit antecedent and hence cannot be pronominalized, it no longer necessarily corresponds to old information, pace Butt \& King's (2000) claim. But Lambrecht \& Lemoine's analysis, according to which the DIO is part of the focus information, might be too strong as well. Rather, I argue that Butt \& King's notion of 'completive information' might be more suitable to describe non pronominalizable DIOs. Furthermore, Lambrecht \& Lemoine overlook another type of non pronominalizable DIOs, which, although less common, also appear in our corpus, namely DIOs that are anaphoric, but receive as antecedent another implicit object rather than an explicit antecedent: although non pronominalizable, these DIOs are closer to the preceding case of pronominalizable DIOs. In each of these cases however, the data remain consistent with our main hypothesis, according to which a verb is informationally prominent over the implicit object: indeed, whether it is topical information or completive information, they remain informationally 
secondary with regard to the focus. Finally, in the case of IIOs, the informational prominence of the verb first and foremost result from the fact that the semantic object is of an indefinite kind : some clues might possibly be inferred from the context regarding the semantic value of the implicit object, but its precise identity does not matter, and it is the verb that bears all the informational weight.

INDEX

Mots-clés : omission de l'objet, proéminence phonologique, proéminence informationnelle, topique

Keywords : object omission, phonological prominence, informational prominence, information structure, topic

\section{AUTEUR}

\section{ANOUCH BOURMAYAN}

University College London, Université Paris 7 Diderot

Anouch.Bourmayan@ens.fr 